\title{
Electron Beam/Converter Target Interactions In Radiographic Accelerators
}

J. McCarrick, G. Caporaso, F. Chambers, Y.J. Chen, S. Falabella,

F. Goldin, G. Guethlein, D. Ho, R. Richardson and J. Weir

This article was submitted to Particle Accelerator Conference 2003, Portland, Oregon, May 12-16, 2003

U.S. Department of Energy

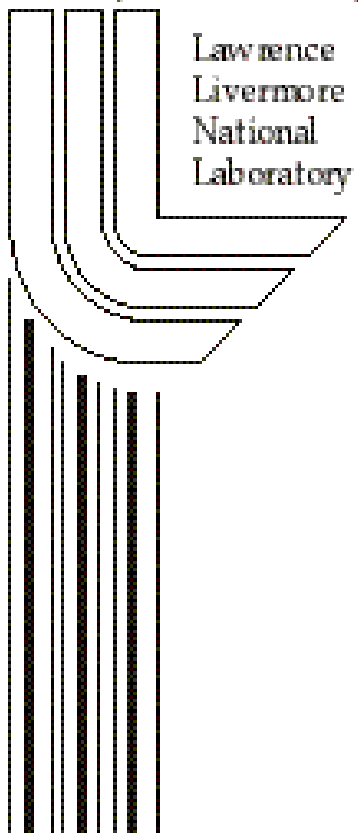


This document was prepared as an account of work sponsored by an agency of the United States Government. Neither the United States Government nor the University of California nor any of their employees, makes any warranty, express or implied, or assumes any legal liability or responsibility for the accuracy, completeness, or usefulness of any information, apparatus, product, or process disclosed, or represents that its use would not infringe privately owned rights. Reference herein to any specific commercial product, process, or service by trade name, trademark, manufacturer, or otherwise, does not necessarily constitute or imply its endorsement, recommendation, or favoring by the United States Government or the University of California. The views and opinions of authors expressed herein do not necessarily state or reflect those of the United States Government or the University of California, and shall not be used for advertising or product endorsement purposes. 


\title{
ELECTRON BEAM/CONVERTER TARGET INTERACTIONS IN RADIOGRAPHIC ACCELERATORS*
}

\author{
J. McCarrick, G. Caporaso, F. Chambers, Y.-J. Chen, S. Falabella, F. Goldin, G. Guethlein, D. Ho, \\ R. Richardson, J. Weir, LLNL, Livermore, CA 94551, USA
}

\begin{abstract}
Linear induction accelerators used in X-ray radiography have single-pulse parameters of the order $20 \mathrm{MeV}$ of electron beam energy, $2 \mathrm{kA}$ of beam current, pulse lengths of $50-100 \mathrm{~ns}$, and spot sizes of $1-2 \mathrm{~mm}$. The thermal energy deposited in a bremsstrahlung converter target made of tantalum from such a pulse is $\sim 80 \mathrm{~kJ} / \mathrm{cc}$, more than enough to bring the target material to a partially ionized state. The tail end of a single beam pulse, or any subsequent pulse in a multi-pulse train, undergoes a number of interactions with the target that can affect beam transport and radiographic performance. Positive ions extracted from the target plasma by the electron beam space charge can affect the beam focus and centroid stability. As the target expands on the inter-pulse time scale, the integrated line density of material decreases, eventually affecting the X-ray output of the system. If the target plume becomes sufficiently large, beam transport through it is affected by macroscopic charge and current neutralization effects and microscopic beam/plasma instability mechanisms. We will present a survey of some of these interactions, as well as some results of an extensive experimental and theoretical campaign to understand the practical amelioration of these effects, carried out at the ETA-II accelerator facility at the Lawrence Livermore National Laboratory.
\end{abstract}

\section{INTRODUCTION}

The purpose of a beam/target system in a radiographic accelerator facility is to produce X-rays from the incoming beam electrons via bremsstrahlung in the target material. Some typical requirements for a single X-ray pulse in an industrial radiography or explosives hydrotesting setting are shown in Table 1. In addition, there are now facilities capable of generating a series of $\mathrm{X}$-ray pulses during a single event, allowing the recording of time-dependent data. The typical time scales of such events are microseconds, so that the pulse spacing in a multi-pulse train is perhaps 500 ns. Examples of such systems are the DARHT-II accelerator, a four pulse hydrotest facility now being commissioned at Los Alamos National Laboratory [1], and the ETA/Snowtron facility at Lawrence Livermore National Laboratory, a lower-energy two-pulse system dedicated to accelerator and converter target research and development [2].

If bremsstrahlung conversion were a completely efficient process, the landscape of beam/target interactions

\footnotetext{
*This work was performed under the auspices of the U.S. Department of Energy by the University of California, Lawrence Livermore National Laboratory, under Contract No. W-7405-Eng-48.
}

would already be well-explored territory. However, for every useful X-ray photon produced, a significant amount of waste heat is deposited in the target material. For the systems under consideration, the amount of heat deposited is roughly independent of beam energy and works out to about $80 \mathrm{~kJ} / \mathrm{cc}$ in a target material such as tantalum (Ta), for a single beam pulse with the parameters given in the table. This is equivalent to $9 \mathrm{eV}$ per target atom. Since most solids are bonded with energies of the order of a few $\mathrm{eV}$, this means that a single beam pulse will turn at least some portion of the target material into a plasma with temperatures in the $\mathrm{eV}$ range.

Table 1: System parameters

\begin{tabular}{|l|l|l|}
\hline $\begin{array}{l}\text { System } \\
\text { Requirement }\end{array}$ & $\begin{array}{l}\text { E-beam } \\
\text { Requirement }\end{array}$ & $\begin{array}{l}\text { X-ray } \\
\text { Requirement }\end{array}$ \\
\hline $\begin{array}{l}\text { High photon } \\
\text { energy }\end{array}$ & $\begin{array}{l}20 \mathrm{MeV} \\
\text { endpoint }\end{array}$ & $1-10 \mathrm{MeV}$ \\
\hline $\begin{array}{l}\text { High dose; } \\
\text { Short exposure }\end{array}$ & $\begin{array}{l}\sim 50 \mathrm{kA} \\
\sim 50 \text { pulse }\end{array}$ & $\begin{array}{l}100 \mathrm{~s} \text { of rads } \\
50 \mathrm{~ns} \text { pulse }\end{array}$ \\
\hline High resolution & $\sim 1 \mathrm{~mm}$ focus & $1 \mathrm{~mm}$ source size \\
\hline
\end{tabular}

Since target material in a vapor or plasma state can undergo significant motion on an inter-pulse time scale, the nature of interactions between a beam and target will acquire an explicit dependence on time as well as energy deposited. As the target evolves, a low-density plume of material will develop ahead of the bulk remainder of material. This low-density region can support significant beam/target interactions that can change the beam characteristics before there has been significant X-ray production.

A beam/target interaction is considered "bad" if it affects either of the key radiographic parameters, source size and dose (in this context, there are no interactions that will have a serious impact on beam energy). This translates into interactions that have a significant impact on electron positions or angles, and thus on the positions and angles of the generated X-rays. X-rays from the wrong position will typically increase the effective source size. X-rays emitted at the wrong angle interact with the surrounding environs of the system and ultimately become a source of background noise, rather than useful dose.

\section{SURVEY OF INTERACTIONS}

Let us consider a brief survey of the various beam/target interactions. The primary interactions are, of 
course, bremsstrahlung and small-angle scatter of electrons. The physics of these interactions need not be repeated here [3]; however, a detail worth noting is that in radiographic systems the "useful" photons are generally those emitted within a small angular cone of some preferred direction, hereafter referred to as "forward dose." Small-angle scatter puts a limit on the thickness of target material that will produce forward dose. Additional material may be present, and may have electrons transporting through it, but very few of those electrons will have their radiation cones aimed in the preferred direction.

The most dangerous beam/target interaction in the present context is the creation and extraction of light ions from contaminants in the target material. By disturbing the delicate balance between beam-generated electric and magnetic forces, ions change the beam transport in the target vicinity and are capable of disrupting source size and lowering forward dose. A typical target is a prompt source of ions, since just a few ns of heating brings the target to a temperature $\sim 600 \mathrm{~K}$, which is enough to desorb contaminants on the target surface. The desorbed neutrals are then ionized by the beam. After tens of ns, the bulk target material is heated into the $\mathrm{eV}$ range, and thermal ionization becomes the dominant ion source. Much work has been done recently to investigate the low-temperature mechanisms of ion production; details can be found in [4] and [5].

The hydrodynamic evolution of the vapor- or plasmastate target material is not a direct beam/target interaction, but nevertheless has significant impact on dose and source size. Radial motion of the target moves material out of the electron beam, lowering the integrated line density of material and therefore the dose. Axial motion of the target changes the geometric aspect ratio between small-angle scatter and X-ray production, so that the effective source size increases at fixed incoming beam size. Thus, target motion places a fundamental limit on the multi-pulse performance of a passive target.

There are a host of other interactions, but in the present application they are "benign" in the sense that they do not affect dose or source size. Typically they either make a change to the system that is steady-state relative to the beam flattop, or the interaction length is too small to have a deleterious effect. Backscattered electrons [6] are an example of the former. Secondary electrons that escape from the target are trapped in the negative potential produced by the beam space charge in front of the grounded target; the system reaches steady circulation in a few transit times, $\mathrm{r}_{\text {beam }} / \mathrm{c} \sim$ a few ps. This low-energy electron "fog" changes the beam transport as a function of target thickness, but is compensated via static changes to the transport system for a given target.

The low-density plasma plume ahead of the bulk target material can interact with the beam as the target expands. At densities much lower than those which generate significant $\mathrm{X}$-rays, the plasma will still support macroscopic image charges and currents in response to the beam charge and current. In any beam-plasma system, one must also worry about exciting a broad family of microscopic instabilities. However, the neutral plasma plume is moving at a velocity of order the thermal speed. For Ta at $5 \mathrm{eV}$ this is $\sim 2.3 \mathrm{~mm} / \square \mathrm{s}$, so that even after several $\square \mathrm{s}$ the plume size is $\sim 1 \mathrm{~cm}$. This is too short an interaction length to affect beam transport, even for a beam experiencing either extreme of complete charge or complete current neutralization via macroscopic mechanisms. Any microscopic modes that could be excited would be strongly convective and insignificant over such a short length. This has been verified experimentally, as detailed below.

\section{BACKSTREAMING ION BEHAVIOR}

The extraction of ions by the electron beam space charge from a source close to the target surface has been considered in numerous prior studies [e.g. 7]. However, it will be useful to review some of the basic behavior.

By disrupting the delicate balance between beam space charge and current normally present in beam transport, positive ions produce a strong focusing effect on the electron beam. Ions generated in the region where the beam is focused on a target pinch the beam to a smaller size, and move the location of the new focus upstream, away from the target. The beam size on the target, and the resulting X-ray source size, eventually grow monotonically. In addition, the oscillatory radial motion of the ions within the beam produces a nonuniform charge distribution and therefore nonlinear focusing forces, resulting in beam emittance growth and a corresponding reduction in forward dose.

The strength of the interaction can be characterized by a quantity called the disruption length, $\mathrm{L}_{\mathrm{d}}$ [8].

$$
L_{d}=r_{\text {beam }} \sqrt{\frac{\square \square^{2} I_{\text {Alfven }}}{f I_{\text {beam }}}}, f \equiv \frac{\square_{\text {ion }}}{\square_{\text {beam }}}
$$

The disruption length represents the size of an ion column that brings a beam to the threshold of monotonic spot growth. The envelope of the beam has been overfocused ahead of the target and expanded back to its original spot size at the targe plane. At full neutralization, $\mathrm{L}_{\mathrm{d}}$ in the systems of interest is $\sim 4 \mathrm{~cm}$.

Light ions can exceed $\mathrm{L}_{\mathrm{d}}$ very quickly. For a typical accelerating potential of $300 \mathrm{kV}$, protons reach 0.76 $\mathrm{cm} / \mathrm{ns}$, so that at the end of a 50 ns beam pulse the ion column would be $38 \mathrm{~cm}$ in length. Species with charge-tomass ratios up to that of $\mathrm{O}+$ are dangerous in $50 \mathrm{~ns}$.

The number of ions to fill the disruption channel is quite small. At full neutralization, the number required is

$$
N_{\text {ion }}=\frac{r_{\text {beam }}}{q c} \sqrt{\square \Pi^{2} I_{\text {Alfven }} I_{\text {beam }} f} \square 10^{12}
$$

or what would be produced by ionizing one-tenth of a monolayer of surface contaminants. The number required is less at lower neutralizations because of the longer interaction length involved. 
The effect can be observed experimentally. Figure 1 shows two sets of gated X-ray camera data from the ETAII accelerator. The four frames from left to right shows data at different times within a single pulse, with gates of $10 \mathrm{~ns}$ each, spanning the $40 \mathrm{~ns}$ flattop of the beam. The first row shows a large incoming beam, which has a slower heating rate and lower accelerating potential, moving into the pinch phase of the ion effect by the end of the pulse. The second row shows a tightly focused incoming beam whose fast pinch phase is not captured in these frames, showing only the monotonic growth phase starting very early in time. The ions are beam-generated within a single pulse due to desorption and ionization of surface contaminants.

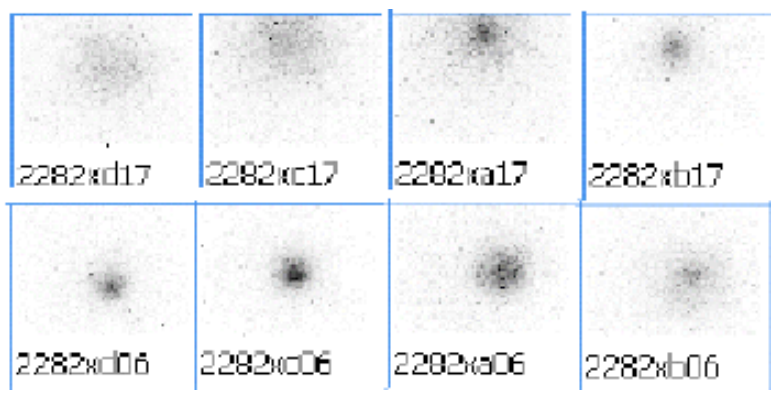

Figure 1: Time evolution of ETA-II X-ray spot with ions, for large (top row) and small (bottom) initial beam sizes.

Figure 2 shows a different form of data. The first plot is a continuous record of beam size versus time taken from a streak camera. Two sets of data are shown, for two different beam sizes. In these experiments, there was a strong ion source present at very early times, arising from a plasma generated very promptly by flashing over the surface of a quartz dielectric in the strong radial electric field of the electron beam. The second plot shows an extremely good match to the data produced via PIC modeling. The time axis of the simulation is shifted relative to the camera data so that the simulation starts at the beginning of the current flattop. It should be noted that the only fitting parameter in the simulation is the choice of ion species, namely $\mathrm{H}+$. While there is much new data that shows $\mathrm{H}+$ does not make up a significant fraction ions in the case of desorption, the quartz flashover data would not be matched by any heavier species.

Given the reality of ion disruption, it is vital to consider various means to combat the effect. The two obvious approaches are cleaning and blocking. In the case of cleaning, one seeks to remove all light contaminants from the target material, generally by some method of heating: resistive, laser ablation, e-beam heating. Such approaches are attractive since they do not interfere with the beam itself, but have undesirable features as well. Since only a small number of ions can cause problems, the cleaning must be very thorough. Furthermore, most approaches require that significant amounts of additional hardware be incorporated into the target system; cleaning must be insitu since the redeposition times for contaminants present in the vacuum system are short relative to the operational times of the experiments.

Blocking means preventing the ions from forming the long interaction channel. This approach, too, has its pros and cons. An approach such as a mechanical barrier can be very simple to implement; however, it interacts with the beam. Both mechanical and electrical approaches (see [9] for examples of the latter) tend to result in trapping rather than absorption of ions, and an ion column too short to disrupt the beam focus can still cause emittance growth.

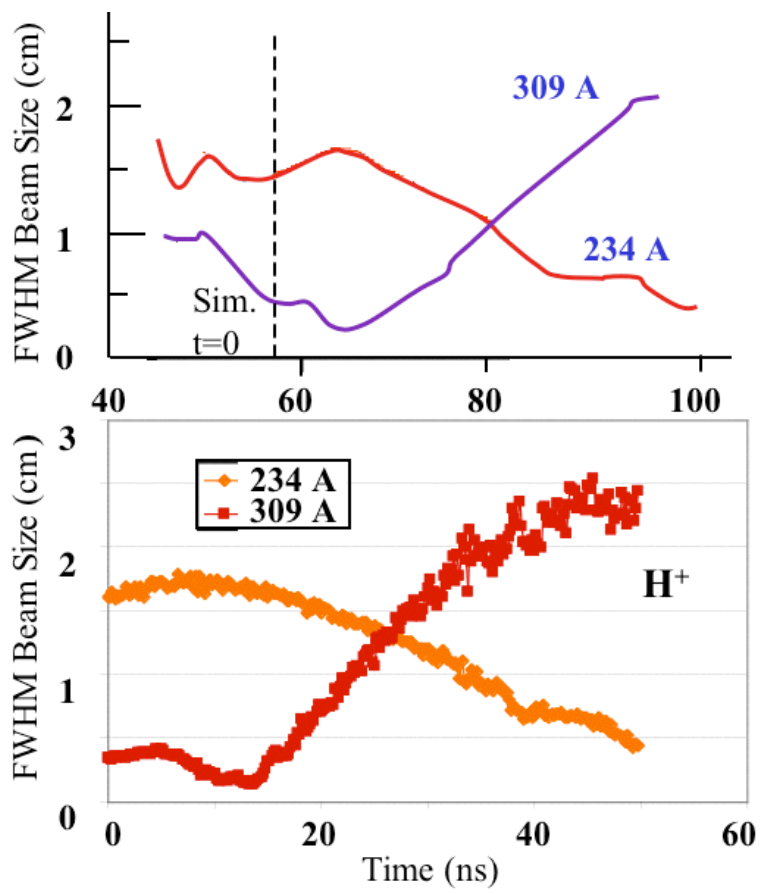

Figure 2: Data (top) and simulation (bottom) of beam disruption by prompt flashover ion source.

\section{TRAPPING IONS WITH A FOIL BARRIER}

The simplest technique for blocking ions is to insert a mechanical barrier, in the form of a thin foil, upstream of the target [10]. In order for the barrier to not be simply another contaminated surface that generates ions, a number of constraints must be met, some of them conflicting:

- The foil must be a grounded conductor. A dielectric will quickly flash over in the radial field of the electron beam.

- $\quad$ The foil must be thick and strong enough for selfsupport, but at the same time thin and light enough that the foil material is not strongly heated by the beam, and does not scatter the beam much in passage. A beam such as DARHT-II can have its emittance doubled by less than $40 \square$ of graphite.

- The foil must be close enough to the target so that the spacing is less than $\mathrm{L}_{\mathrm{d}}$, but at the same time far enough that the beam envelope is large enough to avoid significant heating. 
- Radiographic requirements set the beam size on the target, and the previous requirements set the beam size at the foil and the space between them. This defines a beam envelope, and that envelope must be consistent with the transport system.

Once these requirements are met, the resulting volume defined by the grounded target, grounded barrier, and grounded beam pipe forms a trap rather than an ion absorber. Consider the motion of an individual ion as a function of the $\mathrm{z}$ coordinate between the target and the barrier. If the potential were one-dimensional, this would be a classical turning-point problem where an ion injected at one end with zero energy would reach the opposite end also with zero energy, and reflect. Any finite injection energy would kick the ion from the far end of the trap. In two or three dimensions, however, the problem is completely different. Consider a potential $\square_{0}$ in $(r, z)$ space that is 1-D in $\mathrm{z}$; a trough of infinite radial extent. Superimpose on this a small 2-D "bump" of localized extent, $\square_{1}$, as in Figure 3.

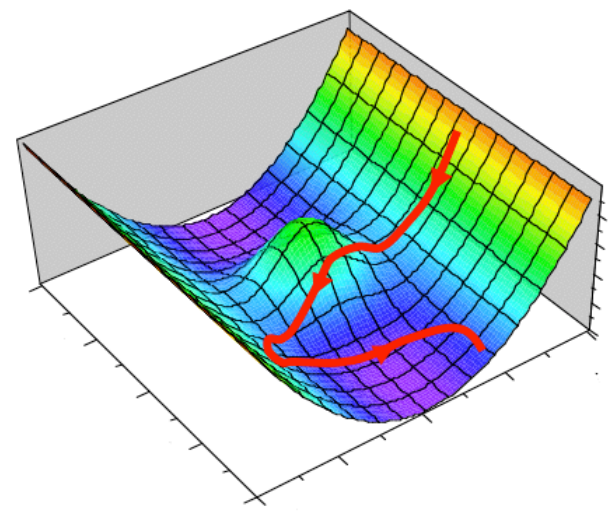

Figure 3: Particle motion in 1-D trough with 2-D bump.

A particle passing over this bump off-center will pick up a "kick" in radial velocity, which for small perturbations is

$$
\square v_{r} \square \frac{\square q}{m} \prod_{z_{\min }}^{z_{\max }} \frac{\frac{\partial \square_{1}}{\partial r} d z}{\sqrt{\frac{\square 2 q}{m} \square_{0}}}
$$

As the particle rises up the far end of the trough, there cannot be any radial forces near the grounded surface that can bring the radial velocity back to zero. There is no path the particle can take back to zero energy, and it will reflect in $\mathrm{z}$ at a critical point defined by

$$
\square\left(z_{\text {crit }}\right) \mid=\frac{1}{2} m \square v_{r}^{2}
$$

This result can be generalized to arbitrarily shaped grounded surfaces, where it can be shown that the bounce near the surface is essentially a specular reflection of the particle.

In the case of an ion in an electron beam, instead of a localized bump there is a long trench, but the mechanism is identical. For an ion oscillating in a uniform, parallel beam, the average kinetic energy tied up in radial velocity near the barrier is

$$
\frac{\square^{r_{\text {emission }}>} \square_{\text {edge }} \frac{r^{2}}{a^{2}} r d r}{\square^{r_{\text {emission }}>} r d r}=\frac{2}{9} \square_{\text {edge }} \square 13 \mathrm{kV}
$$

where $\square_{\text {edge }}$ is the drop from the beam edge to the center, $\sim 60 \mathrm{kV}$. This represents the average injection energy it would take to kick an ion into the barrier, and is clearly greater than any reasonable thermal energy. The trapping effect is robust. This has been verified by fully selfconsistent PIC simulations [11].

The notion of a grounded surface as specular reflector leads to conceptual (not necessarily practical) surfaces that can "bounce" ions completely out of the electron beam. The ions are not energetically trapped within the beam, although they tend to remain so geometrically. Thus, one can modify the geometry to change this behavior. Figure 4 shows one such conceptual surface, along with the beam envelope and tracks of ions that reach considerable radii outside the beam. Such a system could use a dielectric absorber to catch the ions, or simply take advantage of a much slower increase in $\mathrm{f}(\mathrm{t})$, since the volume to be filled becomes the pipe volume and not the beam volume.

Flat barrier systems have been tested successfully in ETA/Snowtron double-pulse experiments [3]. In addition to validating the barrier concept, the experiments also demonstrated safe transport of the electron beam through plasma plumes of various sizes, as the experiment was repeated for various delay times between the Snowtron pulse which generated the plasma and the ETA-II pulse which probed it. Longer times allowed the target/barrier region to fill with larger amounts of plasma; a tight focused was maintained in all cases.

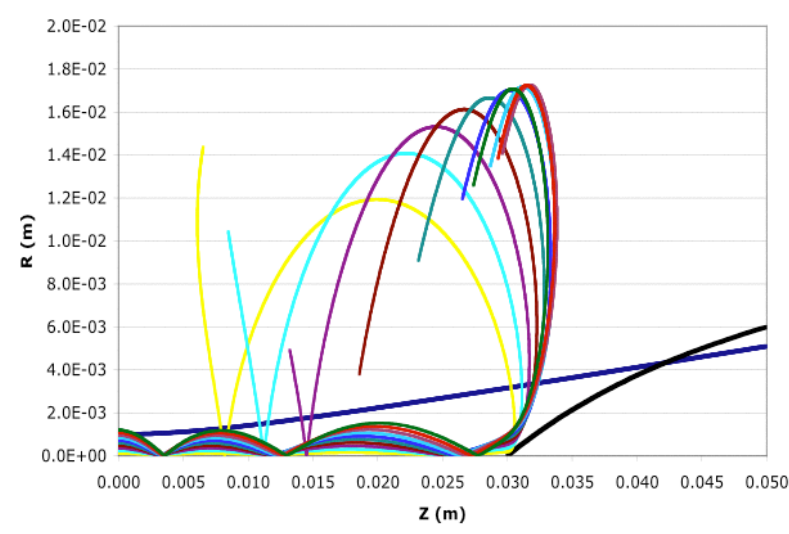

Figure 3: Surface which "bounces" ions out of beam

\section{TARGET HYDRODYNAMICS}

Target motion is the result of beam/target interactions rather than an interaction unto itself, but it is a critical phenomenon. It can be easily shown that, at long times relative to the initial acoustic transit time across the target, the decay of on-axis line density has the form 


$$
\frac{\square(t) d z}{2 \square_{o} d} \square \frac{t_{\text {decay }}^{2}}{t^{2}}
$$

where $\mathrm{d}$ is the target half-thickness and $\square_{0}$ the initial density. The time dependence is independent of the material properties of the target, which enter only through the scale time, $\mathrm{t}_{\text {decay }}$. Let us consider the case of a beam with scale radial dimension $\square$ in the limit of $\square=\mathrm{d} / \square<<1$. One can then show that $t_{\text {decay }}$ has the form

$$
t_{\text {decay }} \square \frac{\square}{\sqrt{C_{o}}} \operatorname{EOS}(1)
$$

where $\operatorname{EOS}(1)$ is an equation of state-dependent factor of order unity, and $\mathrm{C}_{\mathrm{o}}$ is the initial sound speed of the target material just after heating by a beam pulse.

The geometric contribution to $t_{\text {decay }}$ is set by the radiographic requirements and cannot be changed. The initial sound speed is a function of the energy deposition, which is also constrained by the radiographic requirements, and a lumped parameter we will call $\mathrm{E}_{\mathrm{bond}}$. $\mathrm{E}_{\mathrm{bond}}$ is a measure of the energy required per target atom to bring it from the initial solid state to complete vapor, at essentially fixed volume. This is a possible knob to improve target performance.

One approach to using the $\mathrm{E}_{\mathrm{bond}} \mathrm{knob}$ is the composite target concept, described in [12]. The basic idea is build a designer mixture of high-Z material, for $\mathrm{X}$-ray production, and low-Z material, that serves as a heat sink. By building the material with sufficiently small scale size of the high$\mathrm{Z}$ material, one achieves fast heat transfer from high- $\mathrm{Z}$ to low- $Z$ on the time scale of a single beam pulse. The material is designed such that the areal number density of low- $\mathrm{Z}$ material is much higher than the high- $\mathrm{Z}$; the result is to add many more bonds that must be broken before the material can be vaporized, faster than one adds additional beam heating or scatter due to the presence of the additional low-Z material.

Composite targets have been successfully fabricated and tested at the ETA-II facility. Figure 5 compares measurements of $t_{\text {decay }}$ for solid $\mathrm{Ta}$ and a $\mathrm{Ta} / \mathrm{B}_{4} \mathrm{C}$ composite; the measurements were made with a lowenergy X-ray backlighter after targets were struck with the ETA-II beam. The composite material has decay times three times greater than solid $\mathrm{Ta}$ at the tightest beam sizes, increasing to six times greater at more moderate sizes, a significant improvement.

\section{CONCLUSIONS}

Electron beam/converter target interactions in the context of radiography are judged by their effect on the two key radiographic parameters, dose and source size. In general, short interaction lengths protect against all but two interactions: backstreaming ions, and the thermally driven expansion of the target material. While very small amounts of ions can disrupt the beam very quickly, the foil barrier technique has proven to be a simple and effective remedy. Similarly, dramatic decreases in the rate at which line density depletes due to target expansion have been achieved in successful tests of composite target materials.

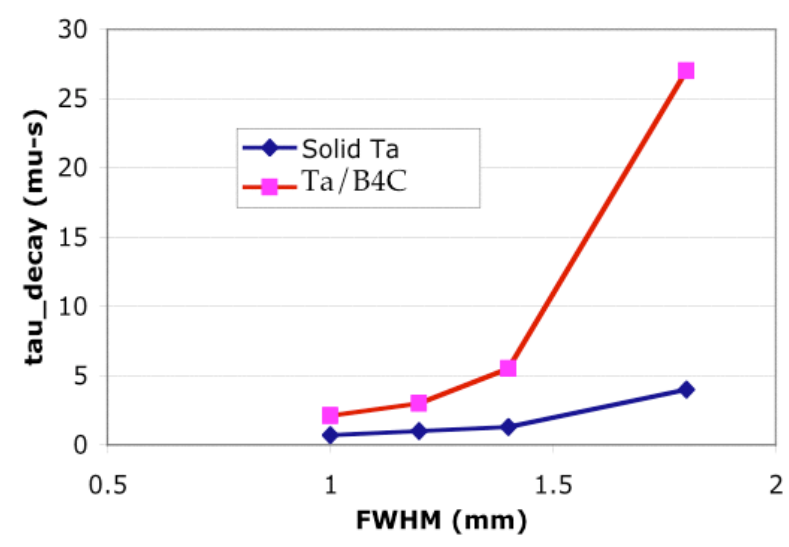

Figure 4: Decay time for composite vs. solid Ta.

\section{ACKNOWLEDGEMENTS}

The authors would like to thank the ETA-II experimental program at LLNL for providing the data shown in this paper.

\section{REFERENCES}

[1] C. Ekdahl, et al. "DARHT-II Commissioning Results," PAC 2003.

[2] S. Sampayan, et. al., "Beam-Target Interaction Experiments for Multipulse Bremsstrahlung Converters Applications", PAC 2001.

[3] J. D. Jackson. Classical Electrodynamics, Wiley, 1962.

[4] T. Hughes, H. Davis. "Effect of Stimulated and Thermal Desorption in DARHT 2," PAC 2003.

[5] H. Davis, et al. "Measurements of Thermally Desorbed Ions from Beam-Target Interactions," PAC 2003.

[6] S. Falabella, et al. "Effect of Backscattered Electrons on Electron Beam Focus," LINAC 2000.

[7] D. R. Welch and T. P. Hughes, Laser Part. Beams 16, 285-294 (1998).

[8] G. Caporaso, Y.-J. Chen. "Analytic Model of Ion Emission from the Focus of an Intense Relativistic Beam on a Target," LINAC 1998.

[9] J. McCarrick, T. Houck. "The Effect of Trapped Backstreaming Ions on Beam Focus in Radiographic Accelerators," PAC 1999.

[10] T. P. Hughes, "Target Calculations for ITS ShortFocus Experiment", MRC/ABQ-N-589, Sept. 1997.

[11] Y.-J. Chen, et al. "High Intensity Beam and X-Ray Converter Target Interaction and Mitigation," AAC 2002.

[12] J. McCarrick. "A Composite Target Concept for Multi-Pulse Radiography," BEAMS 2002. 